Corollary. Suppose that

$$
b b_{h} \ddot{ }+A b \cdot b_{h}+C b \cdot b_{h}=0,
$$

and that $b \propto|t|^{\beta}$ as $b \rightarrow 0, t \rightarrow 0$. Then:

(i) If $C \beta(\beta-1)<0$, almost all $b_{h}(t)$ increase without limit as $t \uparrow 0$ (or $t \downarrow 0$ ).

(ii) If $C \beta(\beta-1)>0$ and $A \beta>1$, then all $b_{\boldsymbol{\alpha}}(t)$ increase without limit as $t \uparrow 0$ (or $t \downarrow 0)$.

(iii) If $C \beta(\beta-1)>0$ and $A \beta<1$, then all $b_{\boldsymbol{h}}(t)$ tend to zero as $t \uparrow 0$ (or $t \downarrow 0$ ).

Proof. Substituting $b=K t^{\beta}$ in (8) and simplifying, we get

$$
t^{2} b_{\dot{h}}+A \beta t b_{\dot{h}}+C \beta(\beta-1) b_{h}=0 .
$$

This has a regular singular point at $t=0$, with the indicial equation

$$
\alpha^{2}+(A \beta-1) \alpha+C \beta(\beta-1)=0 .
$$

The two roots $\alpha_{1}, \alpha_{2}$ of this are

$$
\alpha_{i}=\frac{1-A \beta}{2} \pm \frac{1}{2} \sqrt{(A \beta-1)^{2}-4 C \beta(\beta-1)} .
$$

In Case (i), $\alpha_{1}$ and $\alpha_{2}$ have opposite sign, and so the general solution $b_{h}=c_{1} t^{\alpha_{1}}+c_{2} t^{\alpha_{2}}$ of (8) (logarithmic terms $s^{4}$ do not alter this) increases without limit as $t \uparrow 0$. In Cases (ii)-(iii), $\alpha_{1}$ and $\alpha_{2}$ both have the same sign as $1-A \beta$, whence the conclusions are still obvious.

I wish to acknowledge my indebtedness to Dr. R. L. Ingraham for helpful suggestions about this paper.

\title{
BiBLIOGRAPHY
}

1. A. M. Binnie, The stability of the surface of a cavitation bubble, Proc. Camb. Phil. Soc. 49, 151-5 (1953).

2. H. Lamb, Hydrodynamics, 6th ed., Cambridge University Press, 1932.

3. Sir Geoffrey Taylor, The instability of liquid surfaces when accelerated ..., Proc. Roy. Soc. A201, $192-6$ (1950).

\section{ELECTROMAGNETIC WAVE PROPAGATION IN BOUNDED ELECTRON BEAMS*}

\section{BY PHILIP PARZEN (New York University)}

The linearized Maxwell's equations in $M K S$ units for the field quantities in electron beams are:

$$
\begin{gathered}
\nabla \times \mathbf{E}=-j \omega \mu_{0} \mathbf{H}, \\
\nabla \times \mathbf{H}=\mathbf{J}+\sigma \mathbf{E}+j \omega \epsilon_{0} \mathbf{E}, \\
\nabla \cdot \mathbf{J}+j \omega \rho=0, \\
j \omega \mathbf{V}+\nabla_{0} \cdot \nabla \mathbf{V}+\mathbf{V} \cdot \nabla \mathbf{V}_{0}=\frac{e}{m}\left[\mathbf{E}+\mathbf{V} \times \mathbf{H}_{0}+\mathbf{V}_{0} \times \mathbf{H}\right], \\
\mathbf{J}=\rho_{0} \mathbf{V}+\rho \mathbf{V}_{0} .
\end{gathered}
$$

Received October 20, 1953.

*This work was performed at Washington Square College of Arts and Science, New York University, and was supported in part by Contract AF 19(122)-1, with the U.S. Air Force, through sponsorship of the Air Force Cambridge Research Laboratories, Air Research and Development Command. 
Here all field quantities are the sum of a time independent and a sinusoidally time varying term. E, $\mathbf{H}, \mathbf{J}, \mathrm{V}, \rho$ denote the time varying electric field, magnetic field, electron current density, velocity and charge density, respectively. The subscript " 0 " denotes the corresponding time-independent quantities. $\epsilon_{0}, \mu_{0}, \sigma,-e$ and $m$ are the dielectric constant, permeability, conductivity of the medium, charge and mass of the electron, respectively. The problem is, given the time independent quantities, to solve Eqs. (1)-(5) for the time varying quantities subject to boundary conditions chosen in accordance with the following uniqueness theorem to be proven.

In the case that $\mathbf{J}$ is parallel to $\mathrm{V}$, the time varying field quantities within a surface $S$ are uniquely determined by specifying on the boundary either $\mathbf{E} \times \mathbf{n}$ or $\mathbf{H} \times \mathbf{n}$ and $\mathbf{V}_{0} \cdot \mathrm{V}$ or $\mathbf{J} \cdot \mathbf{n}$. $\mathbf{n}$ is the unit normal to the surface. The proof is as follows: From Eqs. (1) and (2), one gets, denoting conjugates by an asterisk,

$$
\int \mathbf{E} \times \mathbf{H}^{*} \cdot \mathbf{d} \mathbf{S}=j \omega\left[\int\left(\mu_{0} \mathbf{H} \cdot \mathbf{H}^{*}-\epsilon_{0} \mathbf{E} \cdot \mathbf{E}^{*}\right) d \tau\right]+\int \sigma \mathbf{E} \cdot \mathbf{E}^{*} d \tau+\int \mathbf{J}^{*} \cdot \mathbf{E} d \tau .
$$

Using the vector equation identity,

$$
\nabla\left(\mathrm{V}_{0} \cdot \mathrm{V}\right)=\mathrm{V}_{0} \cdot \nabla \mathrm{V}+\mathrm{V} \cdot \nabla \mathrm{V}_{0}+\mathrm{V}_{0} \cdot \nabla \times \mathrm{V}+\mathrm{V} \cdot \nabla \times \mathrm{V}_{0} .
$$

Equation (4) gives:

$$
j \omega \mathbf{V}+\nabla\left(\mathbf{V}_{0} \cdot \mathbf{V}\right)=\frac{e}{m}\left[\mathbf{E}+\mathbf{V} \times \mathbf{H}_{0}+\mathbf{V}_{0} \times \mathbf{H}\right]-\mathbf{V}_{0} \times \nabla \times \mathbf{V}-\mathbf{V} \times \nabla \times \mathbf{V}_{0} .
$$

Since $\mathbf{J}$ and $\mathrm{V}$ and hence $\mathrm{J}_{0}$ and $\mathrm{V}_{0}$ are all parallel to each other,

$$
\mathrm{J}^{*} \cdot \mathbf{E}=\frac{m}{e}\left[j \omega \mathrm{V} \cdot \mathbf{J}^{*}+\mathrm{J}^{*} \cdot \nabla\left(\mathrm{V}_{0} \cdot \mathrm{V}\right)\right] .
$$

Using Eqs. (3) and (5), the following conservation law is obtained:

$$
\begin{aligned}
j \omega\left[\int \mu_{0} \mathbf{H} \cdot \mathbf{H}^{*}-\epsilon_{0} \mathbf{E} \cdot \mathbf{E}^{*}+\frac{m}{e} \rho_{0} \mathbf{V} \cdot \mathbf{V}^{*}\right] & d \tau+\int \sigma \mathbf{E} \cdot \mathbf{E}^{*} d \tau \\
& +\int\left[\mathbf{E} \times \mathbf{H}^{*}+\frac{m}{e}\left(\mathbf{V}_{0} \cdot \mathbf{V}\right) \mathbf{J}^{*}\right] \cdot \mathbf{d} \mathbf{S}=0 .
\end{aligned}
$$

The proof of the uniqueness theorem follows immediately by taking the real part of Eq. (7). This conservation equation can also be used to define impedances and scattering matrices [1]. The condition for oscillations in a resonant cavity is,

$$
\int\left[\mu_{0} \mathbf{H} \cdot \mathbf{H}^{*}-\epsilon_{0} \mathbf{E} \cdot \mathbf{E}^{*}+\frac{m}{e} \rho_{0} \mathbf{V} \cdot \mathbf{V}^{*}\right] d \tau=0 .
$$

The case $\mathrm{J}$ parallel to $\mathrm{V}$ occurs, for example, when $\mathbf{H}_{0}=\infty$. In this case $\mathrm{J}, \mathbf{V}$ and $\mathbf{H}_{0}$ are parallel to each other.

A solution will now be given for the modes of propagation in a right cylindrical waveguide (axis that of the $Z$-axis) which is completely filled with an electron beam which is constrained to move solely along the $Z$ axis $\left(H_{0}=\infty\right.$ and along $Z$-axis) and whose time independent velocity is an arbitrary function of $Z$. According to the uniqueness theorem, the fields in a volume $G$ bounded by the planes $Z=Z_{0}, Z_{1}$, and the waveguide surface is uniquely determined by specifying at $Z=Z_{0} ; \mathbf{E} \times Z$ and $J=E_{s}(0)$ 
and $J(0)$, respectively; and similarly at $Z=Z_{1}$. As in the case without an electron beam [1], the field can be expanded in terms of transverse magnetic $(T M)$ and transverse electric $(T E)$ modes whose coefficients are functions of $Z$. The transverse dependence of these modes are exactly the same as in the space charge-free case. The longitudinal dependence of the $T E$ modes are also the same as in the space charge-free case. The longitudinal dependence of the coefficients of the $T M$ modes is given as follows. Let $J, V, V_{0}, J_{0}, E$ and $H$ denote the longitudinal component of these quantities, and the subscript $Z$ denote differentiation with respect to $Z$. Then, for any mode,

$$
\begin{gathered}
V_{0}^{3} J_{Z Z}+\left[2 j \omega V_{0}^{2}+3 V_{0}^{2} V_{0 z}\right] J_{Z}+\left[2 j \omega V_{0} V_{0 Z}-\omega^{2} V_{0}\right] J=\frac{e J_{0}}{m} j \omega E, \\
E_{Z Z}+\left(k^{2}-T_{1}^{2}\right) E=\frac{j}{\omega \epsilon_{0}}\left(k^{2} J+J_{z Z}\right), \\
-T_{1}^{2} \mathbf{E} \times \mathbf{Z}=-\frac{1}{j \omega \epsilon_{0}} J_{z}-E_{Z}, \\
\mathbf{H} \times \mathbf{Z}=-\frac{1}{j \mu_{0} \omega}\left[(\mathbf{E} \times \mathbf{Z})_{z}-E\right] .
\end{gathered}
$$

$T_{1}$ is the transverse propagation number of the mode and $k^{2}=\omega^{2} \mu_{0} \epsilon_{0}$. The coefficients of the $T M$ modes are uniquely determined by the boundary conditions previously specified which is equivalent to specifying $J$ and $J_{z}+j \omega \epsilon_{0} E_{z}$ at $Z=Z_{0}, Z_{1}$. Equations (9) and (10) constitute a two point boundary condition linear differential system [2] which possesses a unique solution if the system with null boundary conditions has only the trivial solution $J=E=0$. This will be the case according to the uniqueness theorem which implies that zero boundary excitation leads to zero fields in the interior. It is also easy to prove that the fields are uniquely determined by specifying $J, E, J_{z}$ and $E_{z}$ or $J, J_{z}, \mathbf{E} \times \mathbf{n}$ and $\mathbf{H} \times \mathbf{n}$ at $Z=Z_{0}$ and nothing need be specified at $Z=Z_{1}$.

To obtain explicit solutions, Eqs. (9) and (10) are to be solved for $J$ and $E$ subject to initial values of $J, E, J_{Z}$ and $E_{Z}$ at $Z=Z_{0}$. Writing the equations in matrix form,

$$
U_{z}=A U ;
$$

$$
U=\text { column matrix }\left(\begin{array}{c}
J \\
J_{Z} \\
j \omega E \\
j \omega E_{Z}
\end{array}\right) ; \quad U=U\left(Z_{0}\right) \quad \text { at } \quad Z=Z_{0} ;
$$

$$
\begin{aligned}
& \operatorname{matrix} A=\left(\begin{array}{cccc}
0 & 1 & 0 & 0 \\
\gamma_{0}^{2}+j 2 \gamma_{0 z} & -j 2 \gamma_{0}+3 \gamma_{0 z} / \gamma 0 & y^{2} \gamma_{0}^{2} & 0 \\
0 & 0 & 0 & 1 \\
-k^{2}-\gamma_{0}^{2}-j 2 \gamma_{0 z} & j 2 \gamma_{0}-3 \gamma_{0 z} / \gamma_{0} & -k^{2}+T_{1}^{2}-y^{2} \gamma_{0}^{2} & 0
\end{array}\right) \text {; } \\
& \gamma_{0}=\frac{\omega}{v_{0}} ; \quad y^{2}=\frac{e}{\omega^{2} m} \frac{J_{0}}{\epsilon V_{0}} .
\end{aligned}
$$


As suggested by Ramo [3] in the case of $V_{0 z}=0$, a solution is sought which may be expressed as the sum of four exponential functions whose coefficients are uniformly convergent power series in terms of $y^{2}$ and $V_{0 z}$. A convenient method to accomplish this is the matrizant scheme $[2,4]$. The solution is,

$$
U(Z)=\underset{Z_{0}}{\boldsymbol{z}}\{A\} U\left(Z_{0}\right)
$$

$$
\begin{aligned}
& \underset{z_{0}}{\Omega_{0}}(A)=\text { matrizant of } A \\
& \qquad=1+\int_{z_{0}}^{z} A\left(X_{1}\right) d X_{1}+\int_{z_{0}}^{z} A\left(X_{2}\right) d X_{2} \int_{z_{0}}^{x_{1}} A\left(X_{1}\right) d X_{1}+\cdots .
\end{aligned}
$$

This solution is converted to exponential form by transforming $A$ or a portion of $A$ into diagonal form [5]. Only the final result will be given here [6].

$$
U=P(D)\left[P_{1}^{-1}\left(Z_{0}\right)+\Psi P_{1}^{-1}\left(Z_{0}\right)+\phi\left(Z_{0}\right)+\Psi \phi\left(Z_{0}\right)\right] U\left(Z_{0}\right) .
$$

$D$ is a diagonal matrix $=$ diagonal $\left(d_{i}\right), j=1,2,3,4$.

$$
\begin{gathered}
(D)=\operatorname{diagonal}\left(\exp \int_{z_{\bullet}}^{Z} d_{i} d X\right) . \\
U_{m}=\sum_{i}\left[\sum_{k}\left(P_{1}^{-1}\left(Z_{0}\right)+\Psi P_{1}^{-1}\left(Z_{0}\right)+\phi\left(Z_{0}\right)+\Psi \phi\left(Z_{0}\right)\right\}_{i k}\left[U_{k}\left(Z_{0}\right)\right] P_{m i} \exp \int_{z_{\bullet}}^{z} d_{i} d X\right] .
\end{gathered}
$$

The coefficients of the exponentials are uniformly convergent series for small values of $y^{2}$.

Conclusions. A uniqueness theorem for the sinusoidal fields in electron beams has been proved for the case where the current density is parallel to the electron velocity. The modes of propagation in a right cylindrical waveguide completely filled with an electron beam with an arbitrary longitudinal time independent velocity has been worked out.

Acknowledgement. The author wishes to thank Professor Friedman for his aid.

\section{ReFerences}

1. Montgomery, Dicke and Purcell, "Principles of microwave circuits," McGraw-Hill Book Co., Inc., New York, 1948.

2. E. L. Ince, "Ordinary differential equations," Dover Publications, New York, 1944.

3. Simon Ramo, Phys. Rev. 56, 276 (1939).

4. G. Peano, Math. Ann. 32, 455 (1888).

5. H. B. Keller, and J. B. Keller, "On systems of linear ordinary differential equations," New York University, Mathematics Research Group, Research Report No. EM-33.

6. Philip Parzen, "Electromagnetic wave propagation in bounded electron beams," Ph.D. thesis, New York University, June 1953. 